\title{
Interactive comment on "The Pacific Ocean heat engine: global climate's regulator" by Roger N. Jones and James H. Ricketts
}

\author{
Roger N. Jones and James H. Ricketts
}

roger.jones@vu.edu.au

Received and published: 20 November 2020

Thank you for these comments, which we greatly appreciate. Regarding specific points:

[1] Identifying energy flows more clearly

This is a good suggestion and our vagueness reflected a general uncertainty as to which types of energy are involved. We could have been more specific about the nature of heat flows, where shifts in temperature are involved. However, responding to referees' comments has made some aspects much clearer. As the system is complex, we need to consider multiple forms of energy exchange that include dry and most atmospheric heat transfer, sea surface temperatures, and atmospheric feedbacks including cloud feedbacks. Some we can describe with relative confidence, others we 
cannot, but more precise terminology is needed.

[2] More detailed explanations for TWP and TEP being separate reservoirs rather than locations along a flow tube (our rephrasing).

We are sorry if we have given this impression. Even though the paper describes an east-west heat flow, we were at pains to be quite clear that TWP and TEP are distinct entities. The first diagrams are simplified to provide an overall view, but the reality is more complex.

In the responses to the Referees this has been elaborated on and we now have a much better picture of TEP and TWP as distinct entities. The relationship between the two, moving from a cold to hot reservoir up gradient sets up a tension, because if not for the Coriolis effect, movement would be in the other direction. There is two-way exchange between the two on the surface and subsurface, event though the net heat flow is east-west.

We propose that during forced mode (post 1968) TWP acts a thermostat but carries little memory of the heat cycling through it. TEP acts as the more stable node of the two and Granger-type analyses shows that it is the major source of shifts in temperature. It is possible that the two have been close to a critical state since 1968, if not before.

Therefore, in heat engine terms they may be thought of as reservoirs but they also act as nodes within a broader network. If the global climatic network was considered to have a central node, as TEP is the most obvious candidate. Our Granger analysis suggests that TWP acts as the store and TEP the release.

[3] Positive and negative feedbacks acting by definition as to how these contribute to global warming

We don't agree with this definition and have used positive and negative feedback in the discussion paper as to whether processes are amplified or dampened given a specific forcing. The basic structure of both heat engines and attractors in a thermodynamic
ESDD

Interactive comment
Printer-friendly version

Discussion paper 
system requires both positive and negative feedbacks.

Changes to the paper(s)

ESDD

In any revisions, we will take all these points into account, including clarifying [3]. Point [1] will receive particular attention because we consider the other two have been covered under other responses.

Interactive

comment

Interactive comment on Earth Syst. Dynam. Discuss., https://doi.org/10.5194/esd-2019-72, 2019. 